\title{
A Multi-Alert Patient Health Monitoring using Zigbee
}

\author{
Malika \\ Student (M.Tech, EECE) \\ Department of EECE, ITM University, Gurgaon- \\ 122017, India
}

\begin{abstract}
Wireless sensor network is a growing field showing tremendous applications in the areas of medical assistance in hospitals. According to the Medical Statistics, everyday many patients' lives are affected due to negligence in the part of providing immediate care to the patients. Sometimes it is difficult for the staff in the hospitals to race against the time to reach the patient. Solution to this is a need of system that can measure patient health constantly. The main goal of this paper is to alert the staff in the hospitals in case of emergency. This system is designed using sensors and wireless technology with the help of Microcontroller. Sensors will continuously monitor the vital signs until an abnormal condition is detected. After detecting the abnormal condition, alert system comes into action which acts as a multi-alert system.
\end{abstract}

\section{Keywords}

Zigbee, Sensor, GSM, Microcontroller, Health Monitoring.

\section{INTRODUCTION}

The system is a real time intelligent model using multiple sensors on a single system using wireless technology. The system can measure and present the real time activity of the patient without the on-site medical assistance. Wireless Technology used here is Zigbee IEEE 802.15.4 standard. The main advantage of the system is Portability, mobility and reduction in the wiring due to the presence of a low cost wireless Zigbee standard. Zigbee is superior to other existing standards in terms of power consumption, security, network topology and applications. Zigbee have the ability to connect a large number of devices into a single network. ZigBee technology uses the globally available, license-free $2.4 \mathrm{GHz}$ frequency band [8]. The important vital parameter in health monitoring consists of pulse rate, room temperature, humidity, acceleration and smoke. Literature survey indicates that in the hospitals, the main problem leading to increase in the death rate is due to unavailability of doctor or the staff when needed. To resolve this issue, emergency calling switch has been introduced in the system. The patient is required to press the button in case of emergency so that immediate attention is given to the patient. Another important aspect of the patient's health care includes the medicine given to the patient in proper time.

Due to less number of staff as compared to the patients in the hospital, there can be some negligence. Hence, a medicine reminder system is incorporated in the working model. An alarm will be issued, whenever a patient should be given medicine by assigning intervals. In addition, to capture all day data into a notepad file HyperTerminal is used here. This function is included in this system so that Doctor can go through the whole day report and can check for progress in the health of the patient. This paper is organized as follows: System Hardware Design of the system is introduced in Section 2, Section 3 includes the Software Design, Section 4

\author{
Charu Rana \\ Assistant Professor (Sr. Scale) \\ Department of EECE, ITM University, Gurgaon- \\ 122017, India
}

includes Results and Section 5 includes Conclusion part and Future scope.

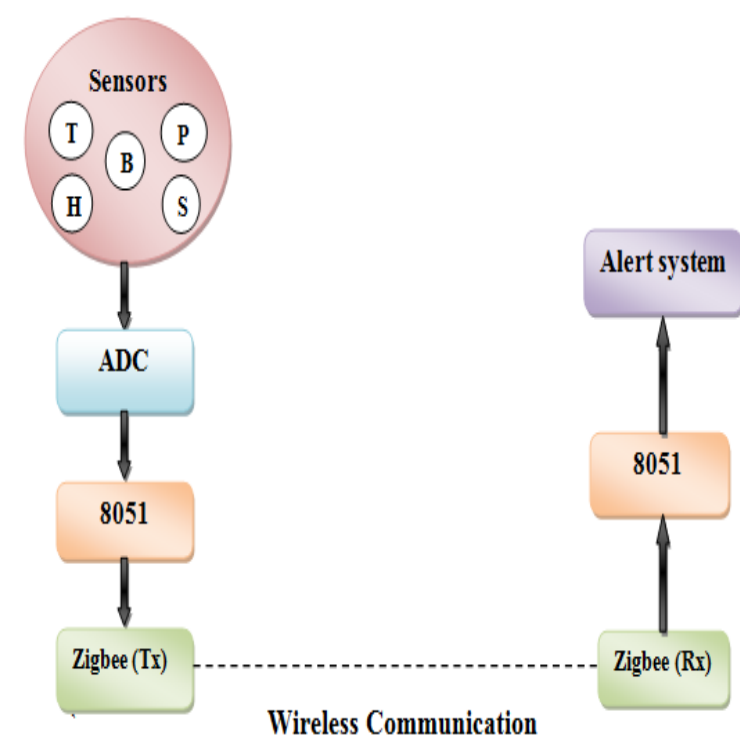

Fig 1: Block diagram of the monitoring system

\section{SYSTEM HARDWARE DESIGN}

To reduce the load of the staff taking care of the patient in the hospitals and to improve patient's mobility, this health care system is designed to compensate for the existing health provisioning system using Zigbee WSN technology. Wireless Sensor Networks has been one of the hottest research topics in the last years. Our main focus is to develop such a wireless system that can raise the standard of health care strategy used now days in the hospitals. The system hardware design is classified into two categories: Transmitter section and Receiver section. The description of the two is as follows:

\subsection{Transmitter section design}

In Transmitter section of the monitoring system five sensors are used. These sensors are $\mathrm{T}$ : Temperature sensor, $\mathrm{H}$ : Humidity sensor, B: Heart beat sensor, P: PIR sensor, S: Smoke sensor. Out of these five sensors, Temperature sensor and PIR sensor is analog thus they are connected to ADC0808 which is an 8-bit A/D converter [11]. All sensors are attached to Zigbee via 8051 microcontroller as the brain of the system as explained in Figure 1 which is the block diagram of the system. Temperature sensors used here is LM35 which is a precise integrated-circuit sensor whose output voltage is linearly proportional to Celsius temperature. For detecting human motion, Pyroelectric sensor Module is used here. It can detect motion up to 6 metres and provide standard active low output. SY-HS-220 is the humidity sensor used here to provide comfort to asthmatic patients. Its function is to measure amount of water molecules in air. MQ2 smoke sensor is to measure combustible gases in the clean air. 


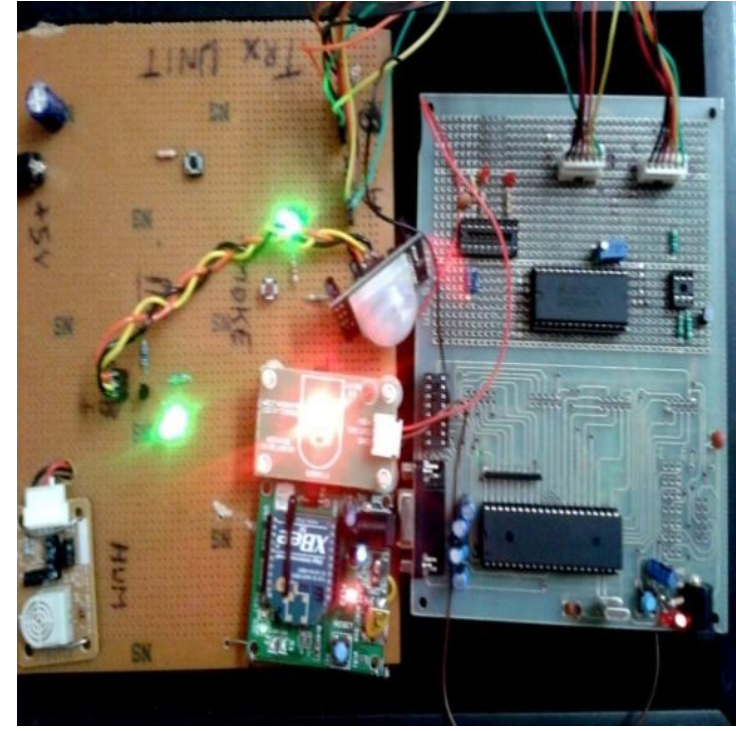

Fig 2: Snapshot of Developed Transmitter section

Lastly, to measure Heart beat in Beats per Minute (BPM) rate, Heart beat sensor by Sunrom Technologies is incorporated here [10]. It's a digital heart rate monitor and is compact in size. All these sensors are the basic requirements of general ward patients so as to provide healthy environment and care to them in hospitals. Then, these sensed data will go to Microcontroller 8051 for further processing before transmit through Wireless Zigbee network. This combination of Sensors, A/D converter, 8051 and Zigbee acknowledge as Transmitter part and will be placed near the patient as shown in figure 2 .

\subsection{Receiver section design}

After the initialization of all the sensors, each of the sensors will continuously check for the data. Each node after obtaining the real time data will send the data to Zigbee via microcontroller. Zigbee is used here in RF module configuration [12]. In receiver part, the sensed real time data will be received by another Zigbee for analysis purpose. The Data received is sent to 8051 for control action. Here limits are defined for each of the sensor node according to the requirements and can be change with the progress in patient. After checking for the limits, status of the patient under supervision will be known. If the data is more than the limits defined, status is Abnormal and if less than or equal to the limits defined, status is Normal.

After this comparison of the values, Alert system comes into action. Alert system includes LCD display, PC display and GSM module. The data from microcontroller will be sent to LCD HDD44780 which is a 14 pin display, Pc display through serial communication and GSM SIM300 modem. For serial communication through RS232 standard, MAX232 IC is used. This IC is used to make RS232 voltage levels compatible with 8051 TTL levels. To receive the data serially and for capturing the real time data on Pc, HyperTerminal is used. Both the abnormal or Normal data will be sending to Pc and LCD unit. The receiver section is placed at the staff level so that they can check for the status of the patient and can take appropriate action.

To alert the doctor, GSM SIM300 modem is used here. As 8051 microcontroller has one UART (Universal Asynchronous Receiver Transmitter) for serial communication which is used here by MAX232 IC for transmitting serial data to Pc. Hence, to have serial communication of 8051 with Sim300 GSM modem, a noninverting buffer/ line driver 74HC244 IC is used here whose output can be directly interface to CMOS, NMOS and TTL. Only the abnormal status will be send to the modem.

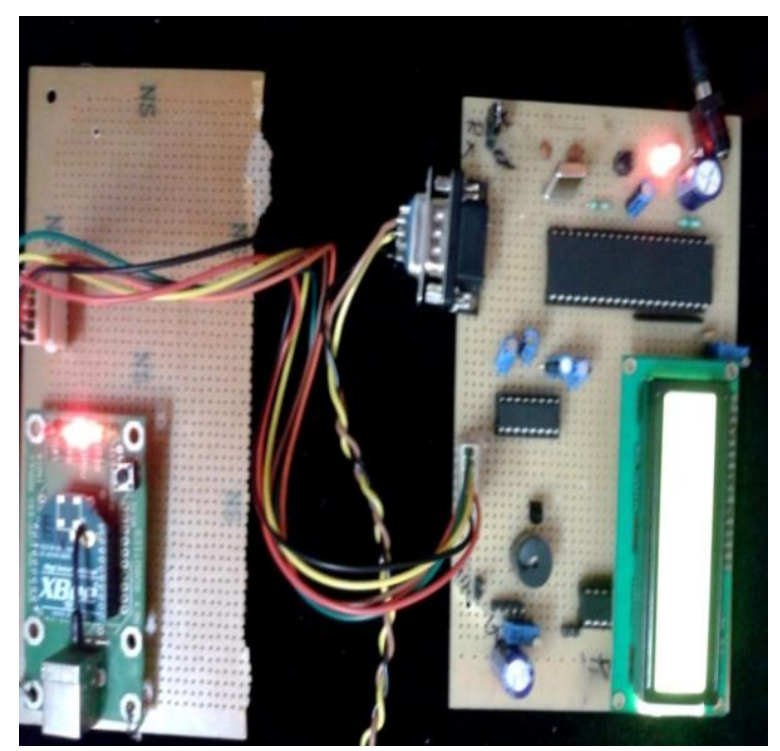

Fig 3: Snapshot of developed Receiver section

This is basically to alert the doctor about the abnormal condition of the patient and needs an immediate supervision. To ensure that there is an emergency, Buzzer alarm is used to alert the staff. Therefore, Zigbee, 8051 and Alert system acknowledge as Receiver section and is placed at the staff corner as shown in figure 3 .

\section{SOFTWARE DESIGN}

The monitoring system is programmed using KEIL $\mu$ vision v8.02 software and programming is done using Embedded C. As in transmitter section Zigbee is programmed in RF module configuration [12], hence logic has been created for each of the sensor used. Software architecture development mainly deals with the receiver section where actual program runs in 8051 microcontroller. The flow chart for the system software development is shown in figure 4 . The initialization process of the system starts by the warm up time requires by the sensors. After that, initialization of ADC0808, 8051, Zigbee, Timers and External interrupt takes place. After the initialization, vital data collection process comes into action. This process goes on until the data is collected at the transmitter section.

After the successful collection of all the vital signs, this data will be forwarded to receiver section wirelessly with the help of Zigbee standard. The receiver section will check for the status of the patient whether normal or abnormal according to the limits defined. If the status is abnormal then the alert signal will be issues for alerting the staff in the hospital and thus better provisioning can be provided to the patient under supervision. But if the status is normal then no alert signal will be issued; only data collected will be shown as results. 


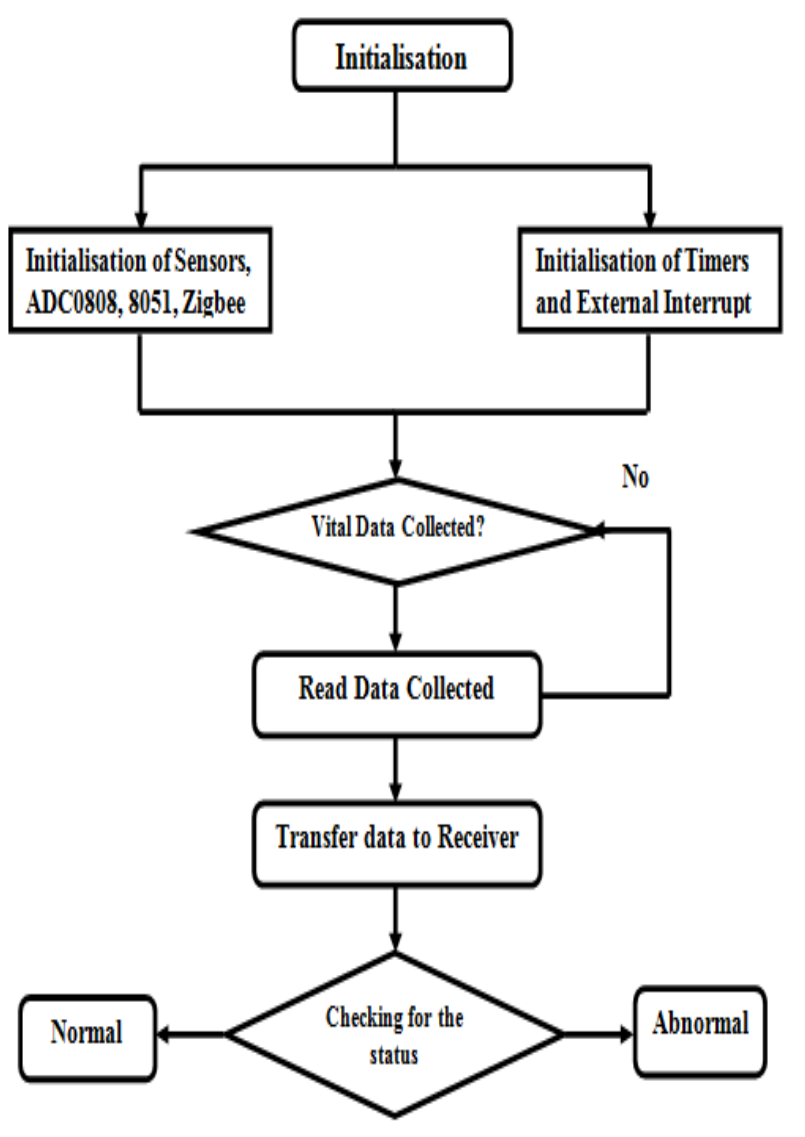

Fig 4: Software Architecture Flow chart

\section{RESULTS}

The sensor network and proposed system were tested in a real time environment. After the collection of real time data by the receiver section and checking for the status of the patient, data will be displayed on alert system. Figure 5 shows the data received by the LCD display.

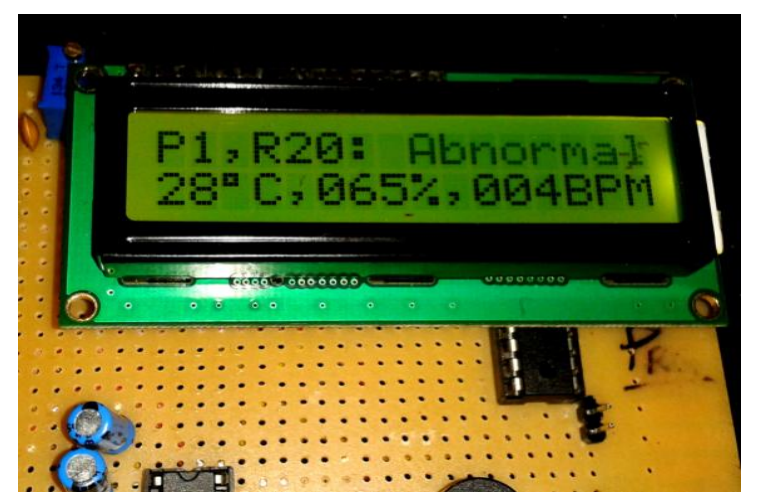

Fig 5: Result for data Received on LCD

Here the message format includes various parameters such as P1 which stands for patient with ID ' 1 ', R20 is for Room number '20', status is abnormal, value of Temperature, Humidity and Heart rate. To assure that data received as obtained by the transmitter, data will also be displayed on Pc using HyperTerminal. HyperTerminal is a window application that is use in order to connect computer with other remote systems.

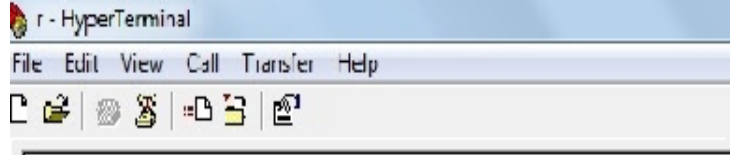

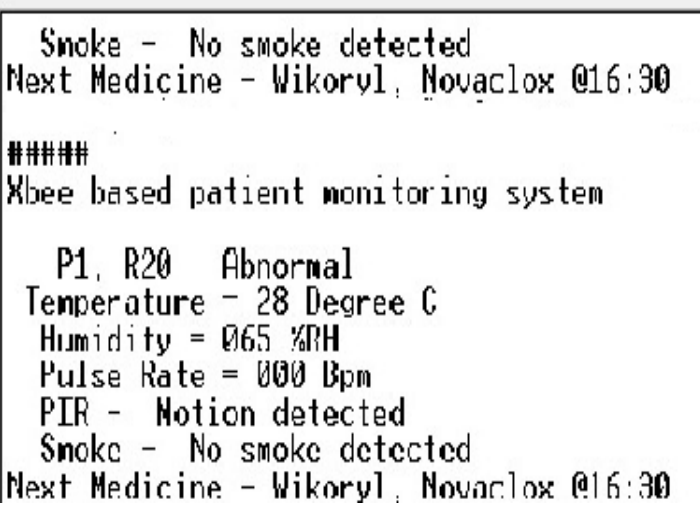

Fig 6: Screenshot of HyperTerminal Window

This application is a useful tool particularly for testing if modem is working properly or for verifying the stable connections with other sites. HyperTerminal can be used to receive or transmit serial data through RS232. For receiving or transmitting serial data with Pc, Data rate, Parity bit, Stop bit and Start bit must be the same as of 8051 microcontroller. Figure 6 shows the data received at HyperTerminal on Pc. Along with the data, Next Medicine reminder will be shown on the HyperTerminal window. In the above HyperTerminal window, the medicines which should be provided to the patients are Wikoryl and Novaclox at 4:30 p.m.

Other function of HyperTerminal includes the capturing of the data received or sent serially. To start capturing data, go to Transfer menu and click on Capture text. The capturing of data will be save as Notepad file as shown in Figure 7 above. Along with the sensed data, medicine reminder, Emergency status will also be shown in HyperTerminal window and Notepad file whenever a patient presses the switch. This notepad file will help the Doctor to check for the progress in patient at the end of the day and take any decision on the basis of this record. Hence this helps in reducing the doctor's burden.

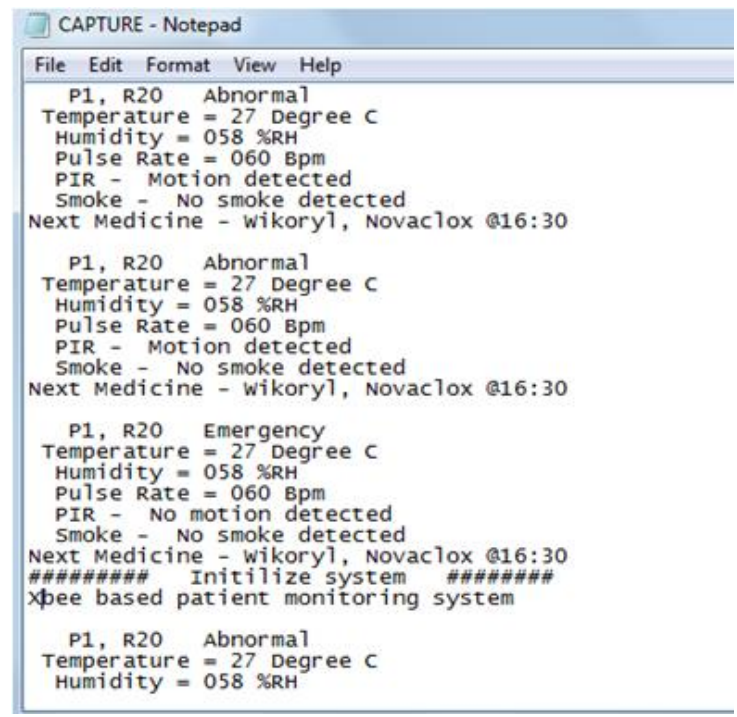

Fig 7: Data Captured as Notepad file 
Also, no matter where the patient is, the doctor will always be connected to monitoring centre via GSM and important messages will be exchanged between them as shown in figure 8 below. Therefore, this multi-alert system keeps on alerting the staff either by displaying the messages on LCD, Mobile phone and the Pc.

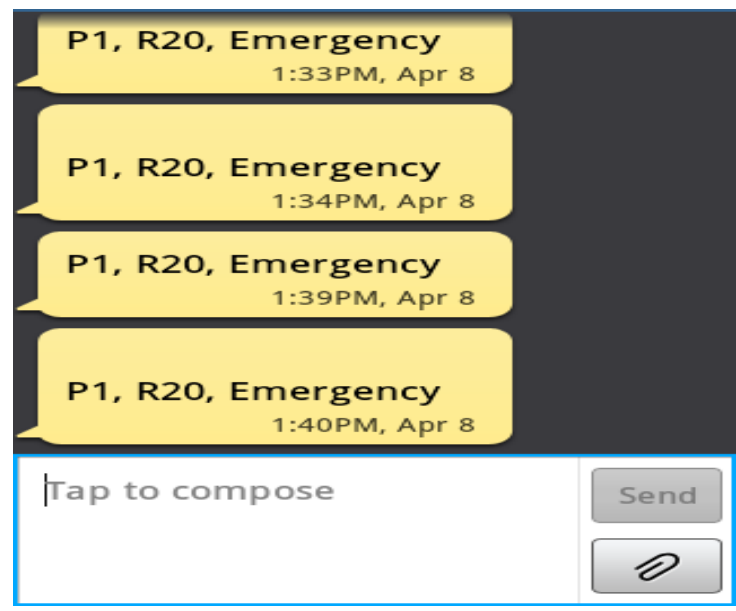

Fig 8: GSM Message Example

\section{CONCLUSION AND FUTURE SCOPE}

In this paper we present a wireless health monitoring system which is able to receive data on receiver side and immediate action will be taken according to the results obtained. This system provides effective solution to upgrade the existing health system by using different kind of sensors mounted on a single system. For example: smoke sensor, humidity sensor, temperature sensor, movement sensor (PIR sensor) and heart rate sensor. This system is based upon wireless technology i.e. Zigbee IEEE 801.15.4 providing low cost effective solution. The system consists of 8051 microcontroller hence we can set values according to the requirement of the patient under consideration. We can modify the parameters with the recovery in the patient. As the parameters can be modified this system is not dedicated to a single patient. Features like patient emergency switch and medicine reminder system will provide better provisioning. Thus the system is convenient and efficient in nature and thus increases interaction between patient and doctor and ultimately avoids unexpected tragedy. This unique combination of Sensors, Wireless standard and Microcontroller will help eliminates the barriers in patient health monitoring and allows the staff and patient to create a healthy relation and thus improved health care. Future scope of this monitoring system includes the addition of more number of sensors and separate health monitoring system for the patients in the Hospital.

\section{ACKNOWLEDGMENTS}

We thank ITM University for providing very conducive research environment and moral support.

\section{REFERENCES}

[1] N.S.A.Zulkifi, F.K.Che Harun and N.S. Azahar, "Centralized Heart Rate Monitoring Telemetry System Using Zigbee Wireless Sensor Network", 2012, IEEEEMBS International Conference on Biomedical and Health Informatics, 265-268

[2] Guojin Li and Jing Han, "Application of the Medical Care System Based on ZigBee Technology", IEEE Symposium on Electrical \& Electronics Engineering (EEESYM), 2012, 301-303

[3] Hongzhou Yu and Lu Liu, "Remote Health Monitoring System Using Zigbee and GPRS Transmission Technology", Fourth International Symposium on Computational Intelligence and Design, IEEE, 2011, $151-154$

[4] A. Sagahyroon, F. Aloul, A.R. Al-Ali, M.S. Bahrololoum, F. Makhsoos and N. Hussein, "Monitoring Patients' Signs Wirelessly', $1^{\text {st }}$ Middle East Conference on Biomedical Engineering”, 2011, 283-286

[5] Chai Jihong, "Patient Positioning System in Hospital Based on Zigbee", International Conference on Intelligent Computation and Bio-Medical Instrumentation, 2011, 159-162

[6] K.Becher, C.P.Figueire, C.Muhle, R.Ruff, P.M.Mendes and K .P. Hoffmann, "Design and Realization of a Wireless Sensor Gateway for Health Monitoring", 32nd Annual International Conference of the IEEE EMBS, 2010, 374-377

[7] Ming Xu, Longhua Ma, Feng Xia, Tengkai Yuan, Jixin Qian and Meng Shao, "Design and Implementation of a Wireless Sensor Network for Smart Homes", International Workshop on Mobile Cyber-Physical Systems (MobiCPS 2010), in conjunction with UIC2010, IEEE, 2010, 239-243

[8] ZigBee Wireless Sensor Applications for Health, Wellness and Fitness, 2009, Zigbee Alliance.

[9] Reza Sahandi and Yuanlong Liu, "Channel Overlap Problems of Zigbee Networks for Remote Patient Monitoring on General Hospital Wards", International Conference on Communications and Mobile Computing, IEEE, 2010, 259-263

[10] Heart Beat Sensor. http://www.sunrom.com/files/1157datasheet.pdf

[11] ADC0808 http://www.nexrobotics.com/images/downloads/ADC080 8.pdf

[12] XBee/XBee-PRO OEM RF Modules. http://www.nexrobotics.com/images/downloads/Manual_ xb_oem-rf-modules_802.15.4.pdf. 